\title{
THE SPOTTED CONTACT BINARY SS ARIETIS - SPECTROSCOPY AND INFRARED PHOTOMETRY
}

\author{
P.P.RAINGER, S.A. BELL AND R.W. HILDITCH \\ Department of Physics and Astronomy, University of St. Andrews, North Haugh, \\ St. Andrews, Fife, KY169SS, Scotland.
}

\begin{abstract}
The first infrared photometry for the W-UMa system SS Ari is presented. An analysis based on medium resolution spectroscopy presented here shows that SS Ari is a W-type system with a mass ratio of 0.33 . It seems certain that the asymmetry in the published light curves and those obtained for this study can be explained by the effect of spots on one or possibly both components of the system. The precise location, size and temperature of these spots require the use of Doppler Imaging techniques in conjunction with high quality multi-band photometry.
\end{abstract}

Key words: stars - binaries: eclipsing - binaries: spectroscopic - infrared photometry - spotmodelling

\section{Spectroscopy and Radial Velocities}

The spectroscopic observations of SS Ari were obtained by PPR during the period 1987 November 8-11 at the Observatorio del Roque de los Muchachos, La Palma, with the 2.5-m Isaac Newton telescope, the intermediate dispersion spectrograph (IDS) and GEC CCD detector. The Jobin-Yvon 1200 grating was employed in conjunction with the $500-\mathrm{mm}$ camera of the IDS to provide spectra at a dispersion of $16.7 \AA \mathrm{mm}^{-1}$. Each spectrum was centred on $4200 \AA$ with a useful range of $\approx 200 \AA$. The integration times for the programme star spectra were typically $1000 \mathrm{~s}$ ( $<3 \%$ of the orbital period) allowing data to be obtained at a signal-to-noise of $\approx 25-30$ while maintaining adequate time resolution.

Preliminary reductions of the spectroscopic data were made via the STARLINK software package FIGARO. The spectra were linearized, rectified and finally loglinearized using REDUCE (Hill, Fisher \& Poeckert 1982) in preparation for the crosscorrelation analysis using vCROss (Hill 1982). Cross-correlation analyses of the spectra of SS Ari were made against HD 693 (Sp. type F6V). A mass ratio of 0.33 has been determined from the six double-lined spectra.

\section{Infrared Photometry and Reductions}

Simultaneous infrared and optical photometry of SS Ari was obtained by RWH in the period 1987 November 19-21 using the United Kingdom Infrared Telescope with the UKT6 and VISPHOT photometers respectively. The comparison star used for these observations was $\mathrm{BD}+23^{\circ} 277$ which has also been used by Katużny\& Pojmański(1984) in their optical study of SS Ari. The observational technique and method of data reduction have been described elsewhere (Bell et al. 1990). Due to an instability in the optical path of VISPHOT, the $B$ and $V$ data were unuseable, however the $J$ and $K$ data obtained with UKT 6 were unaffected by this problem. The $J$ and $K$ light curves are shown in Fig. 1 . 


\section{Ephemeris}

Although a rigorous analysis of the $(O-C)$ data cannot be justified due to the scatter in the photographic and visual data, a sinusoidal variation characterised by a period of approximately $43 \mathrm{yr}$ and an amplitude of 0.036 day can be inferred. If this behaviour could be attributed to third body motion in an orbit coplanar with that of SS Ari, then the total mass of the system can be estimated at $0.13 \mathrm{M}_{\odot}$. As the total mass of SS Ari is approximately $1.6 \mathrm{M}_{\odot}$, this explanation can be rejected unless the orbit of the third body is nearly perpendicular to that of SS Ari.

If the photoelectrically-derived data are examined in isolation, another interpretation can be made. A quadratic function can be fitted to these residuals which could then be attributed to mass transfer from the more massive component at a rate of approximately $2.0 \times 10^{-8} \mathrm{M}_{\odot} \mathrm{yr}^{-1}$. Kurpińska-Winiarska and Zakrzewski (1990) have suggested that there may be some confusion in early time-of-minimum observations caused by the mis-identification of minima. If this is the case then the sinusoidal nature of the $(O-C)$ data is no longer applicable and the simple quadratic function due to mass transfer becomes a more acceptable explanation.

\section{Photometric analysis}

An analysis of the available photometric data for SS Ari suggests a spectral type of G2 and a temperature for the primary component of $5900 \mathrm{~K}$ can be inferred using the tabulations of Popper (1980) and Koornneef (1983).

The $V$ light curve of Kałużny\& Pojmański and the UKIRT $J$ and $K$ data were analysed with the light curve synthesis program LIGHT2 (G. Hill, private communication), an expanded version of LIGHT (Hill 1979). Given the asymmetric nature of the light curves, each half of the $V, J$ and $K$ curves was analysed separately. If the solutions are to be believed then the initial analysis indicates that SS Ari has moved from a marginal contact system with unequal temperature components indicated by the $V$ band data to a system in deep contact with thermalised components indicated by the infrared data. Although the optical and infrared data were obtained in 1982 and 1987 respectively, it is difficult to conceive of a mechanism that could cause such a drastic change to occur on such a short timescale.

If the solutions to the first half of the light curve data are taken to represent the true geometry of the system, then a cool spot visible at second quadrature must be invoked to explain the different quadrature brightnesses. On the other hand, if the solutions to the second half of the light curve data are deemed to be correct, then a hot spot visible at first quadrature must be invoked. If the existence of a hot spot resulting from some form of mass transfer is to be believed, then the deep contact, equal temperature solution suggested by the infrared solutions seems inappropriate. However, the marginal contact, unequal temperature solution suggested by the optical data does provide a system configuration in which such a hot spot might, conceivably, occur.

For the purposes of this analysis, the longitude of a spot is defined as the angle between the sub-stellar point and the spot centre measured anti-clockwise from the sub-stellar point as seen from the north pole of the star. The spot itself is 
characterised by its radius and a temperature which is expressed as an excess over the photospheric temperature of the component on which the spot is located. If the solution to the second half of the $V$ light curve is adopted as the most likely configuration of SS Ari, the observed $V$ light curve can be matched by generating a hot spot on the cooler component. The assumption was made that the spot has the same temperature as the secondary component on the grounds that the feature may be the result of mass transfer in the sense secondary component to primary component. This direction of mass transfer is opposite to that inferred by the possible interpretation of the times of minima $(O-C)$ diagram. However, if mass transfer did occur from the cooler primary component to the hotter secondary component, it is not clear whether a hot spot would be formed. If the system is truly a marginal contact system, with both components filling their respective Roche lobes, then gas streams and associated accretion hot spots (e.g. Hilditch \& King 1986) would not occur. Geometrical considerations indicate that such a spot would have a longitude of approximately $30^{\circ}$ with a radius of some $30^{\circ}$. Similarly, the infrared data require a spot located at approximately $90^{\circ}$ with a radius of some $50^{\circ}$ located on the cooler component. A plausible solution to all three light curves can be obtained by increasing the inclination of the system from $75^{\circ}$ to $78^{\circ}$ and generating a spot of radius $50^{\circ}$ whose longitude is $20^{\circ}$ for the $V$ data and $90^{\circ}$ for the infrared data. The notion of a hot spot at $20^{\circ}$ seems almost acceptable, notwithstanding our above-referenced lack of knowledge of mass transfer mechanisms; but the existence of such a spot at a longitude of $90^{\circ}$ seems extremely difficult to support.

On the other hand, if the first half of the $V$ light curve is assumed to be indicative of the true geometry of the system, then a cool spot must be invoked to explain the depression of second quadrature. The assumption has been made that the spot is on the cooler, primary component as this star is more likely to support spot activity of the type seen on the sun. Extending the solar analogy a little further, the temperature of the spot has been assumed to be $2000 \mathrm{~K}$ cooler than the surrounding photosphere. Consequently, the position and size of the spot were varied to obtain the best match to the $V$ light curve. A spot longitude of $260^{\circ}$ and radius $12^{\circ}$ provides the required depression of second quadrature. Generating $J$ and $K$ curves using this geometry and including a spot at the location determined for the $V$ curve exhibit minima whose depths are too shallow by up to $0 \cdot 1$. Assuming the geometry is correct and the temperatures of the two components are also correct, two further cool spots are required to improve the fits to the minima. One spot has to be placed on the back of the primary component to make primary minimum deeper and a further spot has to be placed on the back of the secondary component to make secondary minimum deeper. It became clear during the generation of many light curves that the sizes of the spots required were different for the $J$ and $K$ curves to obtain good matches to the observed data. For the $J$ curve, two spots of radius $17^{\circ}$ on the primary component were required at longitudes of $180^{\circ}$ and $270^{\circ}$ and also a spot on the secondary component of radius $30^{\circ}$ at a longitude of $180^{\circ}$. For the $K$ curve spots of radius $23^{\circ}$ and $25^{\circ}$ on the primary component were required at longitudes of $180^{\circ}$ and $270^{\circ}$ respectively and also a spot on the secondary component of radius $52^{\circ}$ at a longitude of $180^{\circ}$. These models are shown in Fig. 1. 


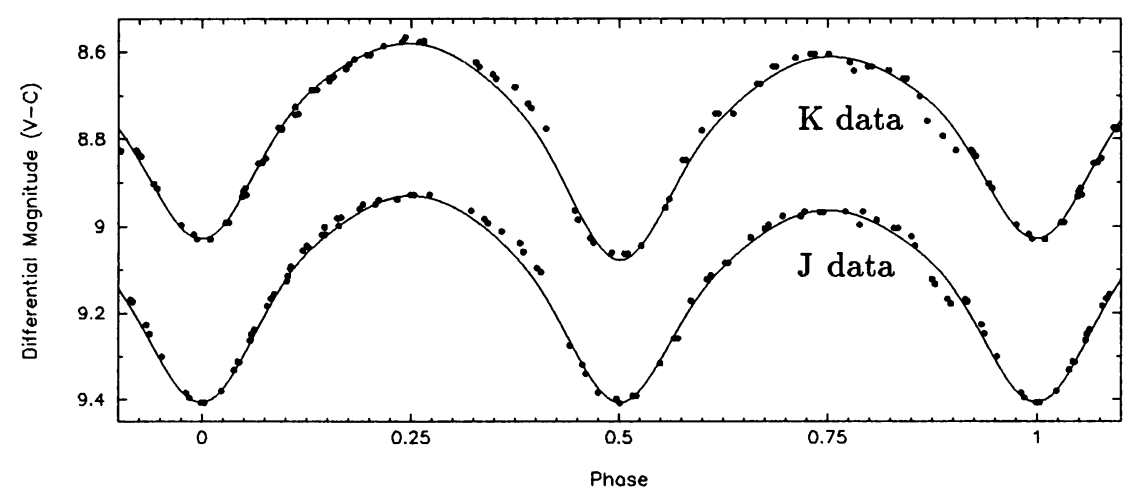

Fig. 1. Spot modelling of the infrared light curves of SS Ari.

Adjusting the temperature excess of the spots did not yield any improvement in the consistency of spot sizes between the $J$ and $K$ curves. It is conceivable that the size and position of a spot may have changed in the interval time between the acquisition of the optical and infrared photometry but it is difficult to explain the difference in the spot sizes between the simultaneous infrared light curves. One possibility is that the temperature distribution over the surface of the spot is more complicated than the simple model used in this analysis. It is also worth pointing out that the spot placed on the back of the secondary component could have been positioned on the facing hemisphere of the primary component although the latitude of the spot would have to be around $30^{\circ}$. This could be indicative of active belts similar to those observed on the Sun. A more complete discussion of this work is given by Rainger et al. (1991).

\section{References}

Bell, S.A., Rainger, P.P., Hill, G. \& Hilditch, R.W.: 1990, Mon. Not. R. astr. Soc. 244, 328

Hilditch, R.W. \& King, D.J.: 1986, Mon. Not. R. astr. Soc. 223, 581

Hill, G.: 1979, Publs. Dom. astrophys. Obs. 15, 297

Hill, G.: 1982, Publs. Dom. astrophys. Obs. 16, 59

Hill, G., Fisher, W.A. \& Poeckert, R.: 1982, Publs. Dom. astrophys. Obs. 16, 43

Kałużny, J. \& Pojmański, G.: 1984, Acta Astr. 34, 445

Koornneef, J.: 1983, Astr. Astrophys. 128, 84

Kurpińska-Winiarska, M. \& Zakrzewski, B.: 1990, Inf. Bull. Var. Stars 3485, 1

Popper, D.M.: 1980, Ann. Rev. Astr. Astrophys. 18, 115

Rainger, P.P., Bell, S.A. \& Hilditch, R.W.: 1991, Mon. Not. R. astr. Soc. In press 